\title{
Nanoparticle Enhanced Laser Induced Breakdown Spectroscopy for Improving the Detection of Molecular Bands.
}

\author{
Can Koral ${ }^{1}$, Alessandro De Giacomo ${ }^{1,2}$, Xianglei Mao, ${ }^{3}$ Vassilia Zorba, ${ }^{3}$ Richard \\ E. Russo ${ }^{3}$
}

${ }^{1}$ Department of Chemistry University of Bari, Via Orabona 4, 70125 Bari, Italy

${ }^{2}$ National Research Council-Institute of Nanotechnology (CNR-NANOTEC), Via Amendola 122/D , 70126 Bari, Italy

${ }^{3}$ Lawrence Berkeley National Laboratory, Berkeley, CA 94720, USA

\begin{abstract}
Enhancement of molecular band emission in laser-induced plasmas is important for improving sensitivity and limits of detection in molecular sensing and molecular isotope analysis. In this work we introduce the use of Nanoparticle Enhanced Laser Induced Breakdown (NELIBS) for the enhancement of molecular band emission in laser-induced plasmas, and study the underlying mechanisms responsible for the observed enhancement. The use of Ag nanoparticles leads to an order of magnitude enhancement for $\mathrm{AlO}\left(\mathrm{B}^{2} \Sigma^{+} \rightarrow \mathrm{X}^{+} \Sigma^{+}\right)$system emission from an Al-based alloy. We demonstrate that the mechanism responsible for the enhancement of molecular bands differs from that of atomic emission, and can be traced down to the increased number of atomic species in NELIBS which lead to AlO molecular formation. These findings showcase the potential of NELIBS as a simple and viable technology for enhancing molecular band emission in laser-induced plasmas.
\end{abstract}

\section{Introduction}

Laser Induced Breakdown Spectroscopy (LIBS) is an optical emission spectroscopy technique that is based on the interaction of an intense pulsed laser beam with a material surface leading to transient plasma formation [1]. While LIBS is largely considered as an elemental analytical technique, the molecular emission also present in the laser induced plasma has received increasing attention in the past few years [2]. Unlike atomic emission lines which are commonly associated with a distinct transition, molecular emission is the result of a group of emission lines from vibrational states originating from electronic transitions of the molecule [3]. The intensity of molecular emission in laser-induced plasmas varies based on the exact molecular transition, the laser parameters, and the background atmosphere.

LIBS molecular band emission has been used in a variety of applications including biological matter [4], complex organic materials such as polymers [5], and explosive residue [6]. Molecular spectra are also the basis for isotopic analysis at atmospheric pressure via Laser Ablation Molecular Isotopic Spectrometry (LAMIS) [7,8]. LAMIS exploits relatively large isotope shifts in spectra of transient molecular isotopologues formed in laser-induced plasmas, thereby enabling isotopic analysis in various material systems in air. Molecular band emission has been also used to elucidate processes in the expanding laser-induced plasma and provide information on complex chemical reactions [3]. 
The use of noble metal nanoparticles (NPs) as spectroscopic enhancers in analytical chemistry is receiving growing interest due to the strong improvements in the analytical performance of these techniques [9-11].The recently proposed Nanoparticle Enhanced LIBS (NELIBS) [10] is an effective method to improve the sensitivity and decrease the Limit of Detection (LOD) in elemental analysis up to two order of magnitude as compared to traditional LIBS of metal alloys. NELIBS is a variant of the LIBS technique, based on the direct deposition of metal nanoparticles on the sample surface. Signal enhancement in NELIBS, is related to the fact that laser pulse induces coherent oscillation of the conduction electrons in metallic NPs and in turn amplifies the incident electromagnetic field, thereby increasing the electric field near the particle surface. This process allows an enhanced production of seed electrons by electron field emission and consequently a more efficient ablation and plasma excitation. Fundamental studies of NELIBS spectra have been previously reported [12], in addition to some examples of analytical applications in the quantitative analysis of metal alloys.

In this work we introduce the use of NELIBS to investigate the plasma emission from molecular species and its effect on the molecular emission intensity. To date, no direct correlation exists between the nanoparticle interactions with the laser pulse and the formation of molecules during the plasma relaxation, due to the fact that the NPs effect mainly occurs during the ablation stage and plasma production [12]. On the other hand, the detection of molecular band emission as a consequence of molecular formation occurs at a late delay time after the laser pulse (microsecond scale in nanosecond plasmas) [1]. We find that molecular emission in NELIBS is much more intense than in LIBS, and interpret this behaviour in the context of molecular formation chemistry and laser-induced plasma dynamics.

\section{Experimental set-up}

An Al-based alloys (NIST: SRM 1255b) was used as a sample for the purposes of this work. Titanium (Ti) and iron (Fe) atoms which are present in the Al-based alloy at concentrations of $0.1477 \%$ and $0.1170 \%$ mass fraction respectively, were used as tracers to study the atomic emission in the laser-induced plasma. The spatial distribution of these species in the Al-based matrix is homogeneous and does not differ spatially to that of Al.

Pure $\mathrm{Al}$ samples were used to experimentally obtain molecular emission from the $\mathrm{AlO}$ $B^{2} \Sigma^{+} \rightarrow \mathrm{X}^{+} \Sigma^{+}$band system and to theoretically fit the corresponding molecular spectra. The $0-0,1-1,2-2,0-1,1-2$ and $2-3$ AlO bands were then assigned up to a rotational number, $J$, of 180 . This information was used to differentiate molecular emission over interfering atomic lines of $\mathrm{Ti}$ and Fe lines which lie in the same spectral windows where $\mathrm{AlO}$ molecular bands are present. Due to the lower concentration of $\mathrm{Ti}$ and $\mathrm{Fe}$ with respect to $\mathrm{Al}$, their atomic emission does not saturate the detector and their lines are not subject to self-absorption.

Within the scope of those investigations, a LIBS apparatus at Lawrence Berkeley National Laboratory, consisted of a laser source for ablation and plasma formation, and a spectrograph for optical emission spectroscopy. A Q-switched Nd:YAG laser (New Wave) with energy of $125 \mathrm{~mJ} /$ pulse and pulse duration of $5 \mathrm{~ns}$ at $1064 \mathrm{~nm}$ was used as the laser source and focused on the target in order to have an irradiance of 1 
GW $\mathrm{cm}^{-2}$. The LIBS spectral emission detection system consisted of a monochromator with a spectral range from 250 to $750 \mathrm{~nm}$ with a 2400 groove $/ \mathrm{mm}$ grating (spectral resolution of $0.2 \AA$ ) (Jobin Yvon Horiba1250M). The monochromator was coupled to an intensified charge coupled device (ICCD) detector (Princeton Instruments, PI-MAX). The laser pulses were focused on the sample by $100-\mathrm{mm}$ focal length lens. The emitted light was imaged onto a fiber optic bundle array using a 300mm-focal-length biconvex UV fused-silica lens. The fiber output was focused on to the monochromator entrance slit by using a UV fused-silica lens doublet.

In order to further study the dynamics of molecular emission, the temporal evolution of the plasma for both NELIBS and LIBS were studied by spectrally and spatially resolved emission images at the Department of Chemistry, University of Bari, Italy. An Ti6A14V grade 5 alloy was used as a sample for these purposes. The LIBS apparatus used throughout those investigations was based on a Q-switched Nd:YAG laser (Quantel Q-Smart) with energy of $850 \mathrm{~mJ} /$ pulse and pulse duration of $6 \mathrm{~ns}$ at $1064 \mathrm{~nm}$, focused on the target in order to have an irradiance of $6.8 \mathrm{GW} \mathrm{cm}^{-2}$. The LIBS emission detection system consists of a monochromator with a spectral range from 250 to $750 \mathrm{~nm}$ equipped with an 1800 groove/mm grating (Jobin Yvon Horiba TRIAX 550), coupled to an ICCD (Jobin Yvon Horiba CCD-3000). A digital delay/pulse generator (Stanford Research Systems, model DG535) was used to synchronize the plasma production and the emission spectra acquisition. The laser pulse was focused on the target with a $100-\mathrm{mm}$ focal length lens. The emitted light was reflected by an aluminum mirror (50-mm diameter) at a $45^{\circ}$ angle with respect to the incident laser, and was focused directly on the monochromator entrance slit using a biconvex UV fused-silica lens triplet. This configuration allowed direct spectrallyresolved imaging of the laser plasma specie distribution.

Prior to each LIBS measurement, the target surface was pre-treated by 5 consecutive laser shots to ensure the surface conditions were kept similar throughout the experiments. The laser spot has been kept of $2 \mathrm{~mm}$ of diameter in all the measurements. In the case of NELIBS, a drop of Ag colloidal solution was deposited on the sample surface. A standardized adjustable-volume micropipette was used to place liquid drops (1 to $8 \mu \mathrm{l})$ of 20 -nm certified spherical Ag NP dispersions $(0.02$ $\mathrm{mg} / \mathrm{mL}$ in aqueous buffer, Sigma-Aldrich) on the target surface. The solution was subsequently evaporated by using compressed air as described in details in Refs. [1112]. In order to optimize the NPs concentration, as usual in the case of NELIBS experiments, we first study the trend of NELIBS/LIBS molecular emission signal intensity ratio as a function of NPs surface concentration as reported in Fig.1.

\section{Results and discussion}

In agreement with Ref [10,12], an evident enhancement of the $\mathrm{Ti}$ and $\mathrm{Fe}$ atomic emission lines, determined as the ratio between NELIBS and LIBS intensity, is shown in Fig.2a for the Al-based alloy. As discussed in previous work, this enhancement is mainly due to the coupling of the laser electromagnetic field with the one induced on NP surface plasmons [11,12]. When this field enhancement occurs, a more efficient ablation and plasma excitation is enabled, resulting in an initial higher excitation 
temperature and in a high number density of emitters which, in turn, causes a strong increase of the emission lines.

On the other hand Fig.2b, shows the comparison between the NELIBS and the LIBS molecular band emission of $\mathrm{AlO}\left(\mathrm{B}^{2} \Sigma^{+} \rightarrow \mathrm{X}^{+} \Sigma^{+}\right.$band system), which is obtained after molecular recombination at a later delay time after the laser pulse irradiation. A high enhancement of the molecular band emission is detected with the NELIBS technique, clearly demonstrating its advantages for the detection of molecular bands. In atomic emission, the NPs show the maximum effect on the enhancement of the optical emission spectra at relatively early times. On the other hand, the formation of molecules in the laser induced plasma occurs much later than the laser matter interaction, and therefore the molecular band emission enhancement cannot be directly explained in connection to the field enhancement.

Molecular bands are formed when electron impact processes are not dominant anymore, such that collisions between the plasma particles, and surrounding background gas start to play an important role [1]. If we consider the formation of $\mathrm{AlO}$ molecules, from a thermodynamic point of view, they are mainly resulting from the following reaction [13]:

$$
\begin{array}{lr}
A l_{(g)}+\mathrm{O}_{2}=\mathrm{AlO}+\mathrm{O} & \text { Eq.la } \\
A l_{(g)}+O+M=A l O+M & E q . l b
\end{array}
$$

The reaction described in Eq.la is spontaneous for all temperature ranges $(\Delta G<0)$, while the reaction of Eq.lb is spontaneous only for T lower than $3000 \mathrm{~K}$ and is in competition with the formation of $\mathrm{O}_{2}$ [13]. For this reason the contribution of Eq.1b can be neglected, so that the rate of $\mathrm{AlO}$ production is given by:

$$
\frac{d[\mathrm{AlO}]}{d t}=k[\mathrm{Al}]\left[\mathrm{O}_{2}\right]
$$

Assuming that the concentration of $\mathrm{O}_{2}$ in Eq. 2 is constant during the entire process, a first order kinetic approximation with respect to $\mathrm{Al}$ is outlined. In this framework, it is reasonable to assume that the enhancement of the molecular band is mainly due to the higher number density of $\mathrm{Al}$ species during NELIBS compared to LIBS. As a matter of fact, during NELIBS, the instantaneous generation of seed electrons by field emission enables multi-point ignition of the plasma and promotes a more homogeneous and efficient ablation. As a result of this, a greater amount of $\mathrm{Al}$ species is excited in to the plasma phase [12]. On the other hand, as a consequence of the different ablation process and of the plasma confinement, in NELIBS, atoms and ions concentrate at shorter distances from the target with respect to the LIBS, in the first few microseconds after the ablation and a much higher number density is observed throughout the temporal evolution of NELIBS. The higher number density achieved with NELIBS can cause a faster decrease of the excitation temperature at this stage, so that, although in the beginning the excitation temperature of NELIBS is higher than that of LIBS, in some hundreds of nanoseconds the situation inverts, as reported in Tab.1. During the plasma evolution, the different transitions are enhanced to a different extent [12]. In order to estimate the actual enhancement, we determined the 
total number density of emitters in NELIBS and LIBS by assuming a Boltzmann distribution for their population at the experimental temperature (Tab.1).

Fig.3 shows typical spectrally resolved emission images and LIBS (a,b) and NELIBS $(\mathrm{c}, \mathrm{d})$ atomic and molecular spectra. This figure shows that initially the plasma spectrum is mainly composed of atomic lines and then the atomic lines are replaced by molecular bands as a consequence of the recombination between atoms of the laser induced plasma and air species accumulated at the plasma front during the plasma expansion. The higher number density of plasma species and the lower temperature during NELIBS suggest a higher production rate of $\mathrm{Al}$ molecules with respect to LIBS. Fig. 4 reports the emission intensity maxima of Ti I line (used as a tracer for atomic species) at $504.00 \mathrm{~nm}$ and the $\mathrm{AlO}$ molecular band system as a function of the distance from the target at different delay times, as obtained by spectrally resolved images.

By inspection of Figs 4 three observations stand out: i) The atomic lines in NELIBS have a longer persistence $(80 \mu \mathrm{s})$ than in LIBS $(50 \mu \mathrm{s})$; ii) In the first tens of microseconds of the plasma evolution, the atomic lines and molecular bands in NELIBS appear to have a different spatial distribution than LIBS, while at later in time they converge to a similar expansion trend; iii) After the atomic emission lines extinguish, the spectra are dominated by molecular bands and no notable difference in the spatial position of the emission band maxima can be observed, but, as in the case of the atomic lines, molecular emission extinguishes later in NELIBS $(250 \mu \mathrm{s})$ than in conventional LIBS $(200 \mu \mathrm{s})$. The latter observation demonstrates that the NPs effect on the LIBS plasma occurs in the beginning of the plasma evolution following which both NELIBS and LIBS plasmas converge to the same equilibrium condition with the surrounding environment. In this view, a similar vibrational and rotational distribution should be expected, although a sharp enhancement of molecular band emission is detected along all the plasma evolution as reported in Fig. 5a and b, where an enhancement of the $\mathrm{AlO} \mathrm{B}^{2} \Sigma^{+} \rightarrow \mathrm{X}^{+} \Sigma^{+}$band system of more than 1 order of magnitude is observed for the most of the plasma persistence time. It is interesting to note that, as shown in Fig.5a, in both LIBS and NELIBS, in the first tens of microseconds, the atomic emission decreases while the molecular band emission increases. Also, as reported in Fig.5b, between 20 and $50 \mu$ s the signal enhancement obtained by NELIBS for atoms and molecules are similar, further confirming the mechanism of formation of $\mathrm{AlO}$ molecule as discussed with Eq. 2. After $50 \mu \mathrm{s}$ molecular band enhancement exceeds atomic, suggesting background gas species also contribute to the enhancement observed in NELIBS. This observation is consistent with more efficient ablation which induces a stronger compression of the surrounding gas, and in turn, a higher number density of air species at the plasma front.

Fig. $6 \mathrm{a}$ and $\mathrm{b}$ show the excitation temperature $T_{e x}$ obtained by the Boltzmann plot of $\mathrm{Ti}$ atoms and the rotational temperature, $T_{J}$ obtained from the AlO molecular band emission during the ablation of the Al- Ti alloy. The corresponding temperatures were calculated using a home-built LabView code that utilizes spectroscopic data from the ExoMol database. As it is shown in Figs $6 a$ and $b$, the difference in both $T_{e x}$ and $T_{J}$ for LIBS and NELIBS is less than $500 \mathrm{~K}$. However, due to the appreciable difference in the energy associated with the electronic transition in atoms and rotational transition in molecules, their effects on the enhancement can be very different. It is important to underline that there is an apparent difference between the data reported in Tab.1 and 
those reported in Fig. 6a. This is because, at later delays from the laser pulse and at large gate widths, the excitation processes in the LIBS plasma lose their efficiency sooner than in the NELIBS plasma, due to the different plasma density in NELIBS and LIBS. Consequently, the integrated time temperature is lower in LIBS than in NELIBS.

As shown in ref.[12] the NELIBS enhancement for atomic spectra is determined as the ratio between the emission intensity of emitting species obtained by NELIBS and LIBS. Within the approximation of Local Thermodynamic Equilibrium (LTE), this enhancement depends on the number density of the species in the plasma and on the temperature, according to the following equations:

$$
\begin{aligned}
\mathrm{R}_{\text {NELIB } / \text { LIB }}^{\text {Atom }} & =\frac{\mathrm{I}_{\text {NELIBS }}}{\mathrm{I}_{\text {LIBS }}} \\
& =\frac{\mathrm{N}_{0, \text { NELIBS }}}{\mathrm{N}_{0, \text { LIBS }}} \frac{\mathrm{Z}\left(\mathrm{T}_{\mathrm{ex}, \text { LIBS }}\right)}{\mathrm{Z}\left(\mathrm{T}_{\mathrm{ex}, \text { NELIBS }}\right)} \exp \left(\frac{\mathrm{E}_{\mathrm{u}}}{\mathrm{k}}\left(\frac{1}{\mathrm{~T}_{\mathrm{ex}, \text { LIBS }}}-\frac{1}{\mathrm{~T}_{\mathrm{ex}, \text { NELIBS }}}\right)\right)_{\text {Eq.3a }}
\end{aligned}
$$

In Eq.3a, $I$ is the experimental spectral intensity, $N_{0}$ is the total number density of the species, $Z(T)$ is the partition function, $E_{u}$ the energy of the upper level, $T_{e x}$ the excitation temperature obtained by the Boltzmann distribution, and $k$ is the Boltzmann constant. For the determination of excitation temperature the following $\mathrm{Ti}$ I lines have been used: 498.173, 499.107, 499.95, 500.721, 501.428, 502.003, 502.287, 503.591, 503.647, 503.84, 503.996, $506.465 \mathrm{~nm}$.

Similarly, the enhancement for the molecular band is expressed as:

$$
\begin{aligned}
& \mathrm{R}_{\mathrm{NELIBS} / \text { LIBS }}^{\text {Molecule }}=\frac{\mathrm{I}_{\mathrm{NELIBS}}}{\mathrm{I}_{\text {LIBS }}} \\
& =\frac{\mathrm{N}_{0, \text { NELIBS }} \mathrm{F}_{\mathrm{e}}\left(\mathrm{T}_{\text {ex }, \text { NELIBS }}\right)}{\mathrm{N}_{0, \text { Lib }} \mathrm{F}_{\mathrm{e}}\left(\mathrm{T}_{\text {ex,LIBS }}\right)} \frac{\mathrm{Q}_{V}\left(\mathrm{~T}_{\mathrm{V}, \text { LIB }}\right) \mathrm{Q}_{\mathrm{J}}\left(\mathrm{T}_{\mathrm{J}, \text { LibS }}\right)}{\mathrm{Q}_{\mathrm{V}}\left(\mathrm{T}_{\mathrm{V}, \text { NELIBS }}\right) \mathrm{Q}_{\mathrm{J}}\left(\mathrm{T}_{\mathrm{J}, \text { NELIBS }}\right)} \cdot \\
& \cdot \exp \left(\frac{\mathrm{E}_{\mathrm{V}}}{\mathrm{k}}\left(\frac{1}{\mathrm{~T}_{\mathrm{V}, \text { LIBS }}}-\frac{1}{\mathrm{~T}_{\mathrm{V}, \text { NELIB }}}\right)\right) \exp \left(\frac{\mathrm{E}_{\mathrm{J}}}{\mathrm{k}}\left(\frac{1}{\mathrm{~T}_{\mathrm{J}, \text { LIB }}}-\frac{1}{\mathrm{~T}_{\mathrm{J}, \text { NELIB }}}\right)\right)_{E q .3 b}
\end{aligned}
$$

where $F_{e}$ is the fraction of molecules in a given electronic state regardless of the vibrational and rotational level, $Q_{v}$ and $Q_{J}$ are the vibrational and rotational partition functions, and $E_{v}$ and $E_{J}$ are the energy of the upper vibrational and rotational levels of the considered transition. $T_{v}$ and $T_{J}$ are the vibrational and rotational temperatures, which under LTE conditions can be considered equal.

For simplicity, Eqs. 3a and b can be considered as the combination of two functions: the first depends just on the ratio of the number density of emitters in NELIBS and LIBS, while the second, that includes the exponential terms and the partition functions, depends on the temperature. Based on this, it is possible to estimate the percentage of contribution to the observed enhancement due to the number density difference between NELIBS and LIBS (which depends on the ablation mass and 
plasma dynamics), and the contribution due to the different temperatures in NELIBS and LIBS plasmas (mainly depending on the electron energy distribution function and plasma dynamics). As shown in Fig. 7a and b, in the case of atoms the effect of temperature is evident as the emission intensity generated from transitions involving high energy levels are more enhanced in NELIBS compared to that associated with low energy levels, as has been demonstrated in detail in Ref.[12].

On the contrary, in molecular band emission in NELIBS, the main contribution is the higher number of molecules formed through the interaction between the plasma and the background gas. As discussed previously, the kinetics of molecular formation are more supported during NELIBS than LIBS due to the greater amount of molecular precursors, i.e. mainly $\mathrm{Al}$ atoms and oxygen species.

\section{Conclusion}

In this paper the effect of nanoparticle deposition on sample surface for improving the emission signal of the AlO molecular band during LIBS of Al-based alloys has been reported. An AlO $\left(B^{2} \Sigma^{+} \rightarrow \mathrm{X}^{+} \Sigma^{+}\right.$band system) emission intensity enhancement of more than 1 order of magnitude has been observed for most of the plasma persistence time. While for atomic species the enhancement is due to the coupling of the ablation laser electromagnetic field with that induced by the surface plasmonic resonance of the NPs, the enhancement of the molecular emission has been ascribed to the higher number of atomic species in NELIBS, which are the main molecular precursor of the AlO molecule formation. To further investigate the phenomenon of NELIBS enhancement, the dynamics of the plasma and the excitation and rotational temperature has been studied in order to outline the contributions of emitters number density and distribution functions temperature for both in the case of atoms and in the case of molecules. It has been found that while for atomic emission the difference in excitation temperature have an important effect on emission enhancement in NELIBS with respect to the LIBS, in the case of molecular emission the main contribution is due to the higher number of emitting molecules, further confirming the effect of NELIBS in the kinetics of formation of molecules produced by the interaction of laser induced plasma species with the surrounding air.

\section{Acknowledgements}

This work at Lawrence Berkeley National Laboratory was supported by the Office of Basic Energy Sciences, Chemical Science Division of the U.S. Department of Energy under contract number DE-AC02-05CH11231.

\section{References}

[1] A. De Giacomo, M. Dell'Aglio, O. De Pascale, V. Palleschi, C. Parigger , A. Wood (2014). Plasma Processes and emission spectra in Laser Induced Plasmas: a point of view. Spectrochimica Acta Part B (2014) 180-188.

[2] A.A. Bol'shakov, X. Mao, J.J. Gonzalez, and R.E. Russo. 2016. "Laser ablation molecular isotopic spectrometry (LAMIS): Current state of the art", Journal of Analytical Atomic Spectrometry 31(1): 119-134.

[3] Á. Fernández-Bravo, T. Delgado, P. Lucena, and J. J. Laserna, "Vibrational emission analysis of the $\mathrm{CN}$ molecules in laser-induced breakdown spectroscopy of organic compounds," Spectrochim. Acta B 89, 77-83 (2013). 
[4] S. J. Rehse, H. Salimnia and A. W. Miziolek, Laser-induced breakdown spectroscopy (LIBS): an overview of recent progress and future potential for biomedical applications Journal of Medical Engineering \& Technology, 2012; 36: 7789.

[5] M. Baudelet, M. Boueri, J. Yu, S.S. Mao, V. Piscitelli, X. Mao, R.E. Russo, Timeresolved ultraviolet laser-induced breakdown spectroscopy for organic material analysis, Spectrochim. Acta Part B, 62 (2007), pp. 1329-1334

[6] J. L. Gottfried, F. C. De Lucia Jr, C. A. Munson, A. W. Miziolek, Laser-Induced Breakdown Spectroscopy for Detection of Explosives Residues: A Review of Recent Advances, Challenges, and Future Prospects, Anal. Bioanal. Chem. 2009, 395, 283

[7] R.E. Russo, A.A. Bol'shakov, X. Mao, C.P. McKay, D.L. Perry, and O. Sorkhabi. 2011. "Laser ablation molecular isotopic spectrometry", Spectrochimica Acta Part BAtomic Spectroscopy 66(2): 99-104.

[8] H. Hou, G.C.Y. Chan, X. Mao, R. Zheng, V. Zorba, and R.E. Russo. 2015. "Femtosecond filament-laser ablation molecular isotopic spectrometry", Spectrochimica Acta Part B-Atomic Spectroscopy 113(1): 113-118.

[9] F.C. Adams, C. Barbante, Nanoscience, Nanotechnology and Spectrometry, Spectrochimica Acta Part B (2013) 86, 3-13.

[10] A. De Giacomo, R. Gaudiuso, C. Koral, M. Dell'Aglio, O. De Pascale, Nanoparticles Enhanced Laser Induced Breakdown Spectroscopy on metallic samples, Analytical Chemistry (2013) 85, 10180-10187.

[11] A. De Giacomo, C. Koral, G. Valenza, R. Gaudiuso, M. Dell'Aglio

Nanoparticle Enhanced Laser Induced Breakdown Spectroscopy for microdrop analysis at sub-ppm level Analytical Chemistry (2016), DOI: 10.1021/acs.analchem.6b00324.

[12] A. De Giacomo, R. Gaudiuso, C. Koral, M. Dell'Aglio, O. De Pascale, Nanoparticle Enhanced Laser Induced Breakdown Spectroscopy (NELIBS): effect of nanoparticles deposited on sample surface on laser ablation and plasma emission. Spectrochimica Acta Part B, 98 (2014) 19-27.

[13] X. Bai, V. Motto-Ros, W. Lei, L. Zheng, J. Yu, Experimental determination of the temperature range of $\mathrm{AlO}$ molecular emission in laser-induced aluminum plasma in air, Spectrochimica Acta Part B (2014), 99, 193-200.

\section{Figure captions}

Fig.1. Dependence of the signal enhancement of AlO molecular emision intensity, estimated as the ratio between NELIBS and LIBS emission line intensity at the maximum peak of the 0-1 band, as a function of $20 \mathrm{~nm}$ Ag NP surface concentration. Acquisition conditions: Tg: $40 \mu \mathrm{s}$, Td: $40 \mu \mathrm{s}$. Experimental conditions are laser energy $850 \mathrm{~mJ}$, spot size $2 \mathrm{~mm}$.

Fig.2. NELIBS and LIBS optical spectral emission of the a) Ti I and Fe I atomic lines Experimental conditions were: laser energy $850 \mathrm{~mJ}$, spot size $2 \mathrm{~mm}$, delay time $10 \mu \mathrm{s}$, gate width $10 \mu \mathrm{s}$. and b) $\mathrm{AlO} \mathrm{B}^{2} \Sigma^{+} \rightarrow \mathrm{X}^{+} \Sigma^{+}$molecular band. Experimental conditions were: laser energy $850 \mathrm{~mJ}$, spot size $2 \mathrm{~mm}$, delay time $60 \mu \mathrm{s}$, gate width $10 \mu \mathrm{s}$. For NELIBS the Ag-NP (20 nm diameter) surface concentration was $610^{-4} \mathrm{mg} \mathrm{cm}^{-2}$.

Fig.3. Spectrally resolved emission image of LIBS and NELIBS of Al-based alloys and corresponding spectra. Detection delay time was $10 \mu \mathrm{s}$ and $50 \mu \mathrm{s}$, and gate width 
was $10 \mu \mathrm{s}$. LIBS atomic (a) and molecular (c) spectra are shown in black, and NELIBS atomic (b) and molecular emission (d) is shown in red.

Fig.4. NELIBS and LIBS comparison of spatial distribution of emission maxima of Ti I atomic line at $504.00 \mathrm{~nm}$ and $\mathrm{AlO} \mathrm{B}^{2} \Sigma^{+} \rightarrow \mathrm{X}^{+} \Sigma^{+}$band system as a function of delay time as obtained by the spectrally resolved images. Experimental conditions were: laser energy $850 \mathrm{~mJ}$, spot size $2 \mathrm{~mm}$. For NELIBS Ag-NP (20 nm diameter) surface concentration $610^{-4} \mathrm{mg} \mathrm{cm}^{-2}$. Detection time is $10 \mu \mathrm{s}$ of gate width and delay step of $10 \mu \mathrm{s}$.

Fig.5. a) NELIBS and LIBS comparison of emission intensity of atomic line ( $\mathrm{Ti}$ I at $504.00 \mathrm{~nm}$ ) and $\mathrm{AlO} \mathrm{B}^{2} \Sigma^{+} \rightarrow \mathrm{X}^{+} \Sigma^{+}$band system as function of delay time, and b) corresponding atomic and molecular emission enhancement. Experimental conditions are laser energy $850 \mathrm{~mJ}$, spot size $2 \mathrm{~mm}$. For NELIBS Ag-NP (20 nm diameter) surface concentration $610^{-4} \mathrm{mg} \mathrm{cm}^{-2}$. Detection time is $10 \mu \mathrm{s}$ of gate width and delay step of $10 \mu \mathrm{s}$.

Fig.6. Comparison of NELIBS and LIBS temperatures at different delay time from the laser pulse: a) excitation temperature obtained by $\mathrm{Ti}$ I Boltzmann plot and b) rotational temperature obtained from $\mathrm{AlO} \mathrm{B}^{2} \Sigma^{+} \rightarrow \mathrm{X}^{+} \Sigma^{+}$band system. Experimental conditions are laser energy $850 \mathrm{~mJ}$, spot size $2 \mathrm{~mm}$. For NELIBS Ag-NP (20 nm diameter) surface concentration $610^{-4} \mathrm{mg} \mathrm{cm}^{-2}$. Detection time is $10 \mu \mathrm{s}$ of gate width and delay step of $10 \mu \mathrm{s}$.

Fig.7. Percentage contribution factors from Eq. 3 to NELIBS emission signal enhancement a) for atomic species and b) for molecular species. Red bar represents the contribution due to emitter number density and blue bar is the contribution due to the temperature.

\section{Table captions.}

Tab.1. Boltzmann plot analysis of NELIBS and LIBS during titanium ablation at different time delays from the laser pulse and gate width of $500 \mathrm{~ns}$, at laser irradiance of $1.5 \mathrm{GW} \mathrm{cm}^{-2}$ at $1064 \mathrm{~nm}$ (for NELIP $3.510^{-4} \mathrm{mg} \mathrm{cm}^{-2}$ of 20 diameter Ag NPs). The temperature was obtained by the slope of the Boltzmann plot and the relative total number density was estimated by the intercept and the partition function at the experimental temperature, while $\mathrm{K}$ is an instrumental constant. The fraction of ionized emitters was calculated by the Saha equation at the experimental temperature. 


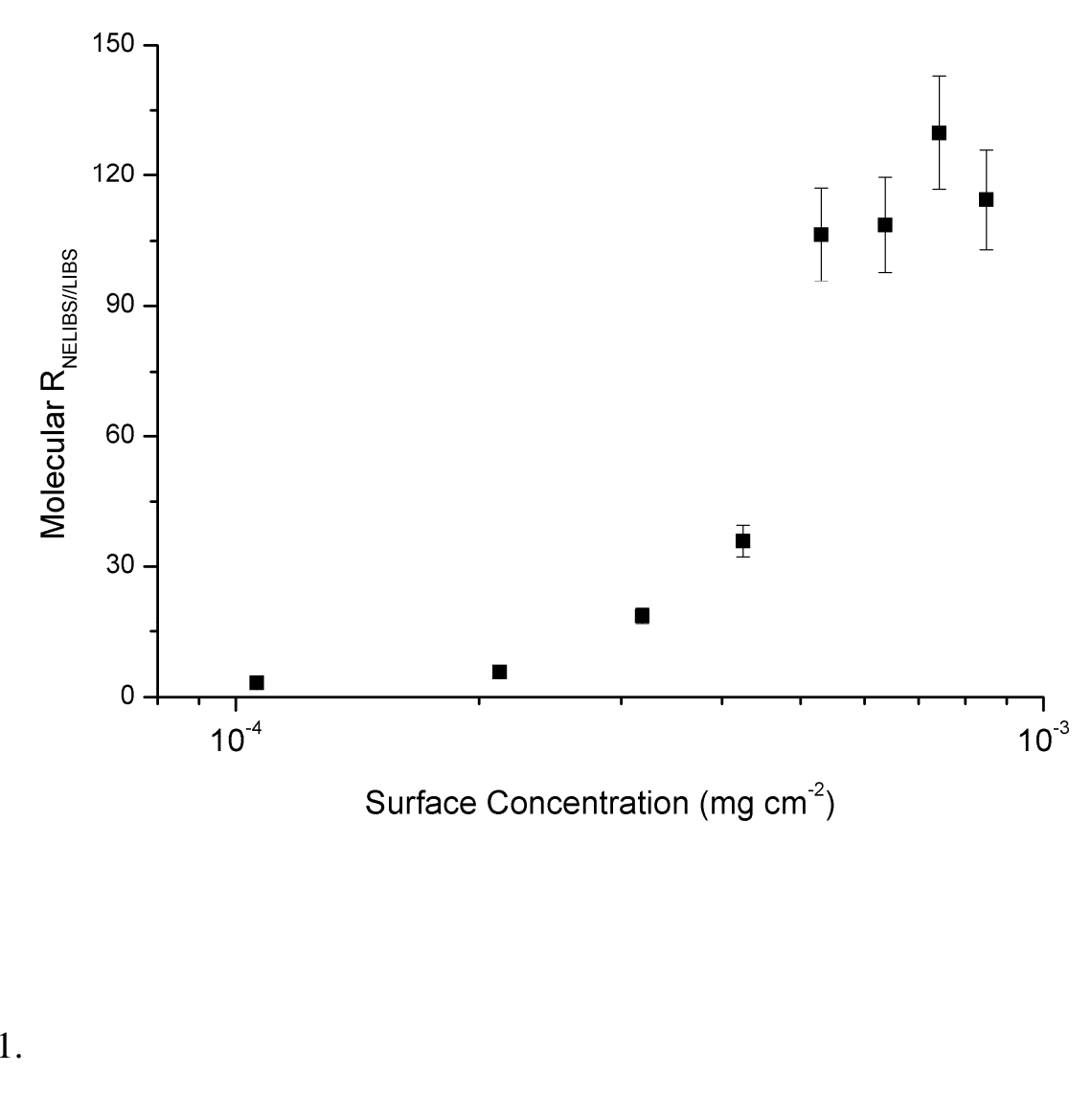

FIG.1.

FIG.1.

Figure(s)

.

the

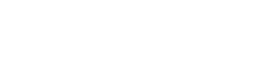

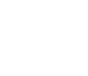




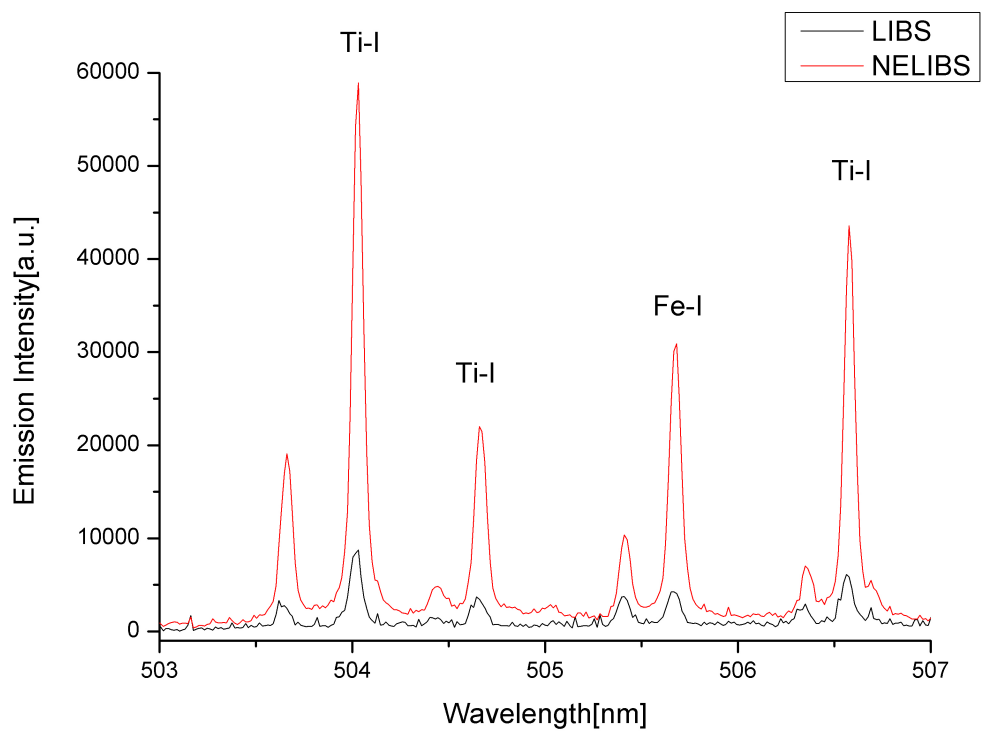

a)

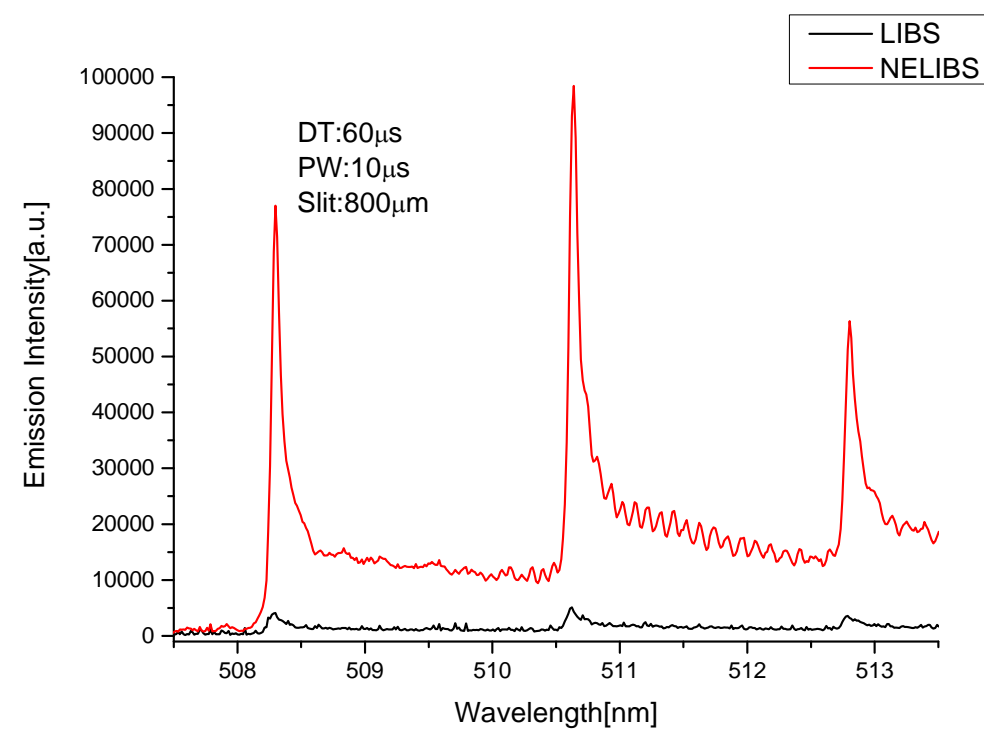

b)

FIG.2a-b 


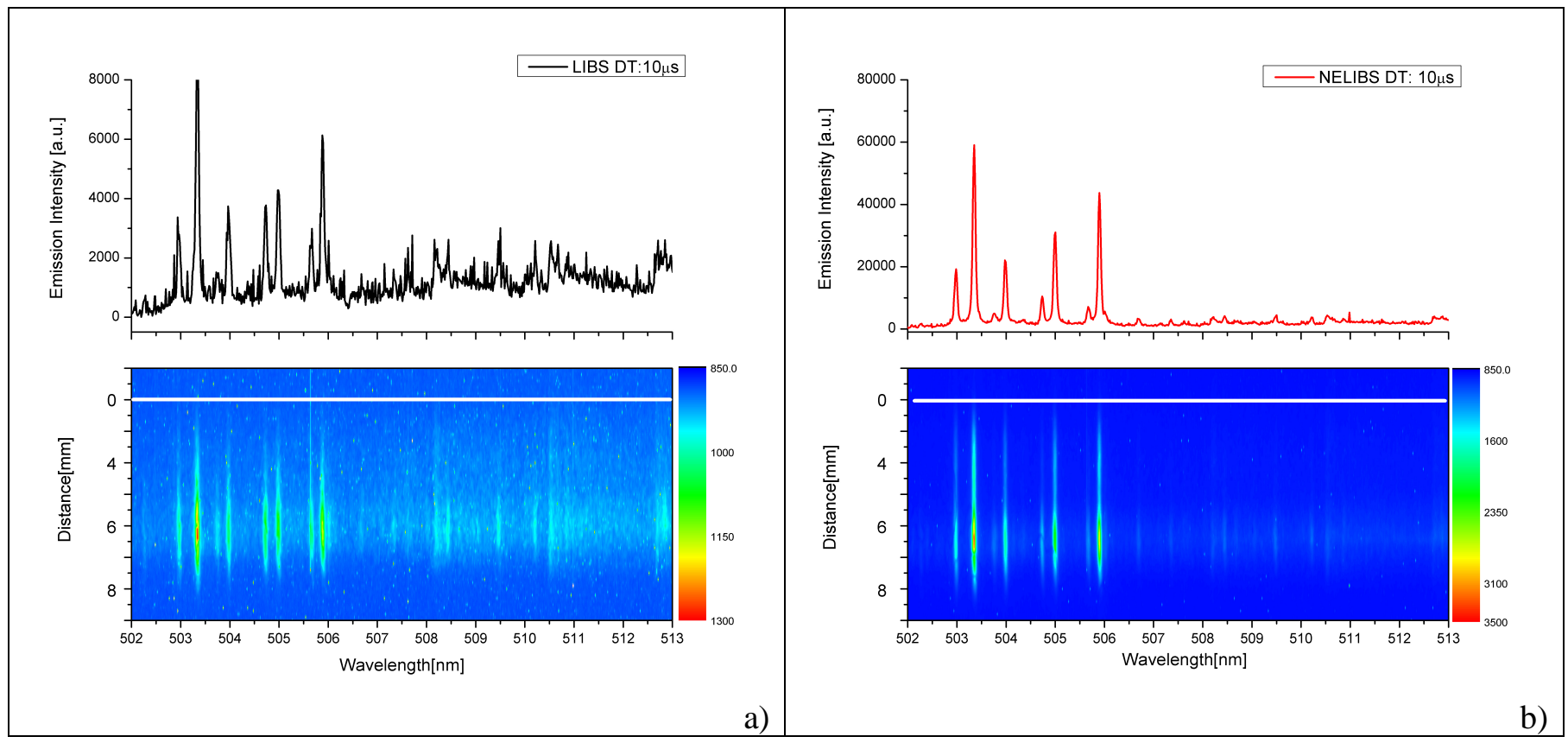




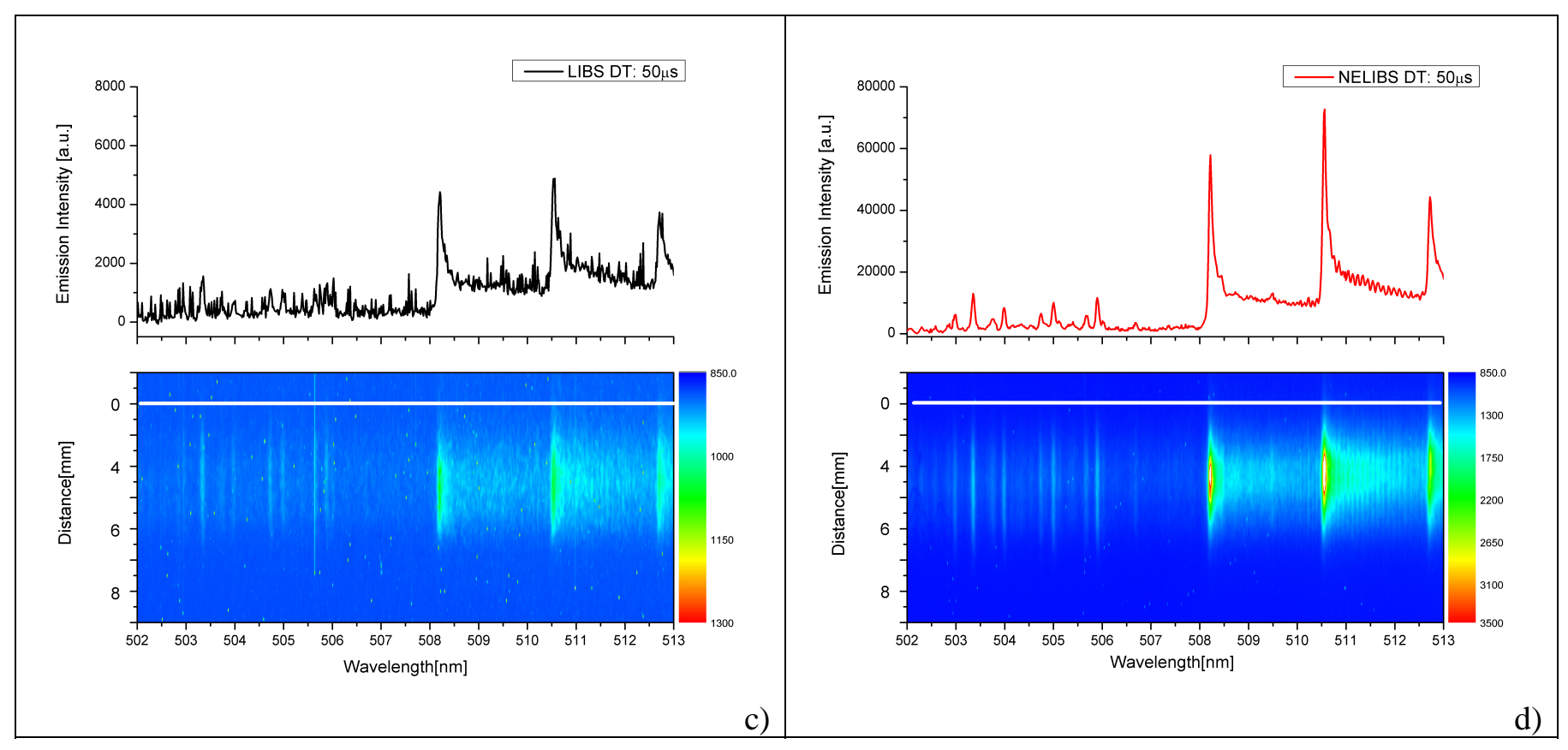

FIG.3 


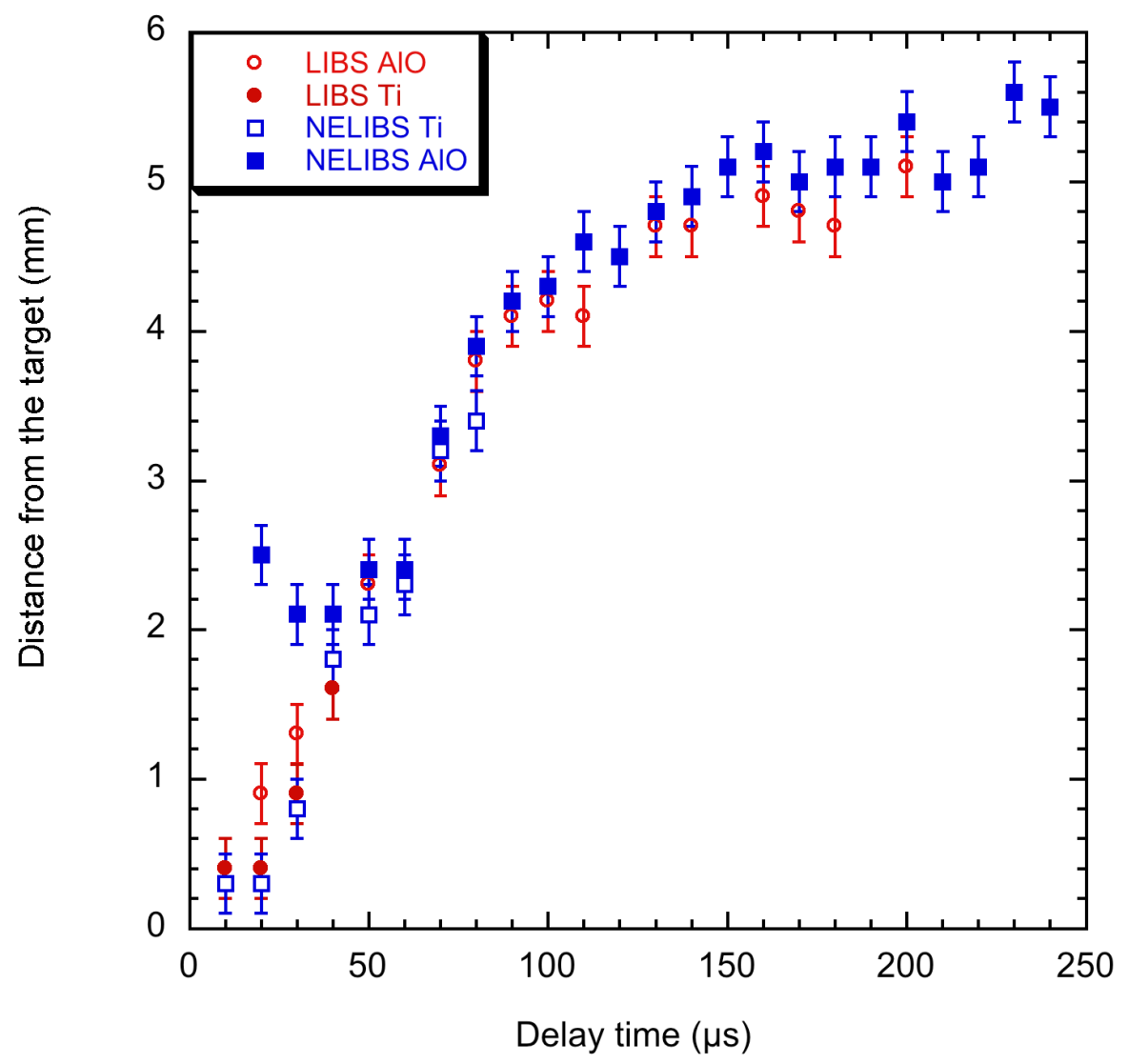

FIG.4 


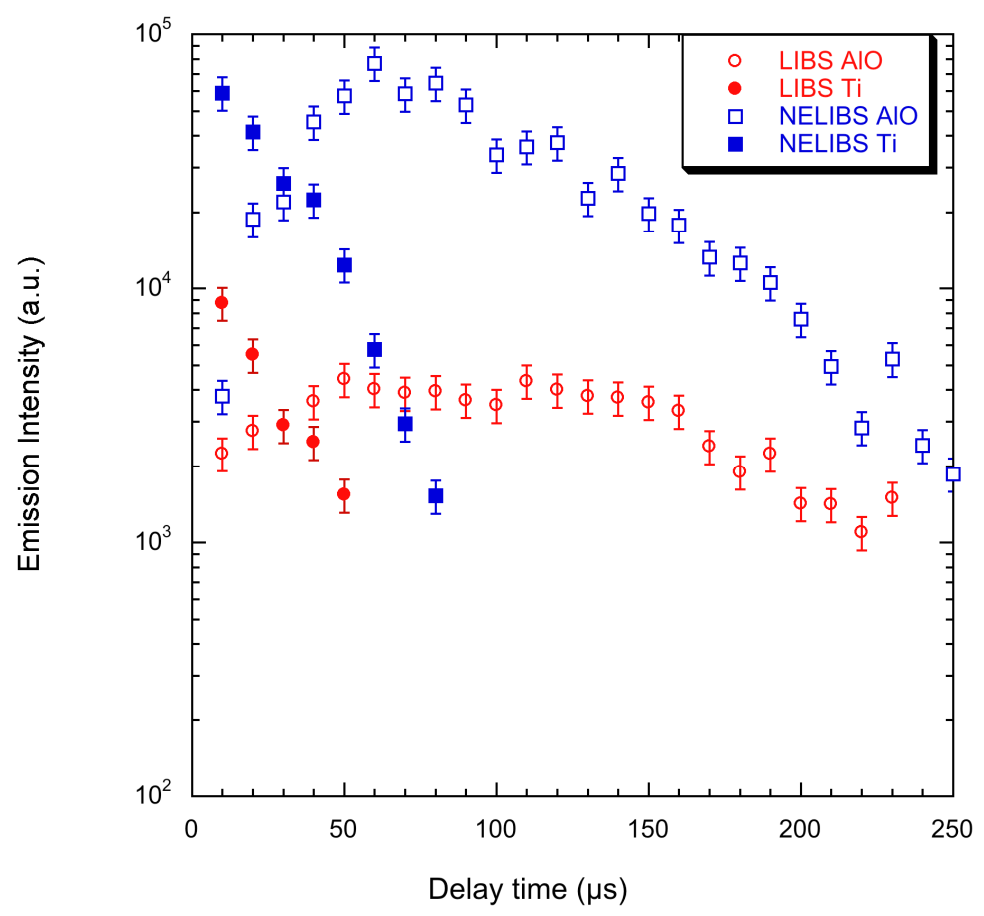

a)

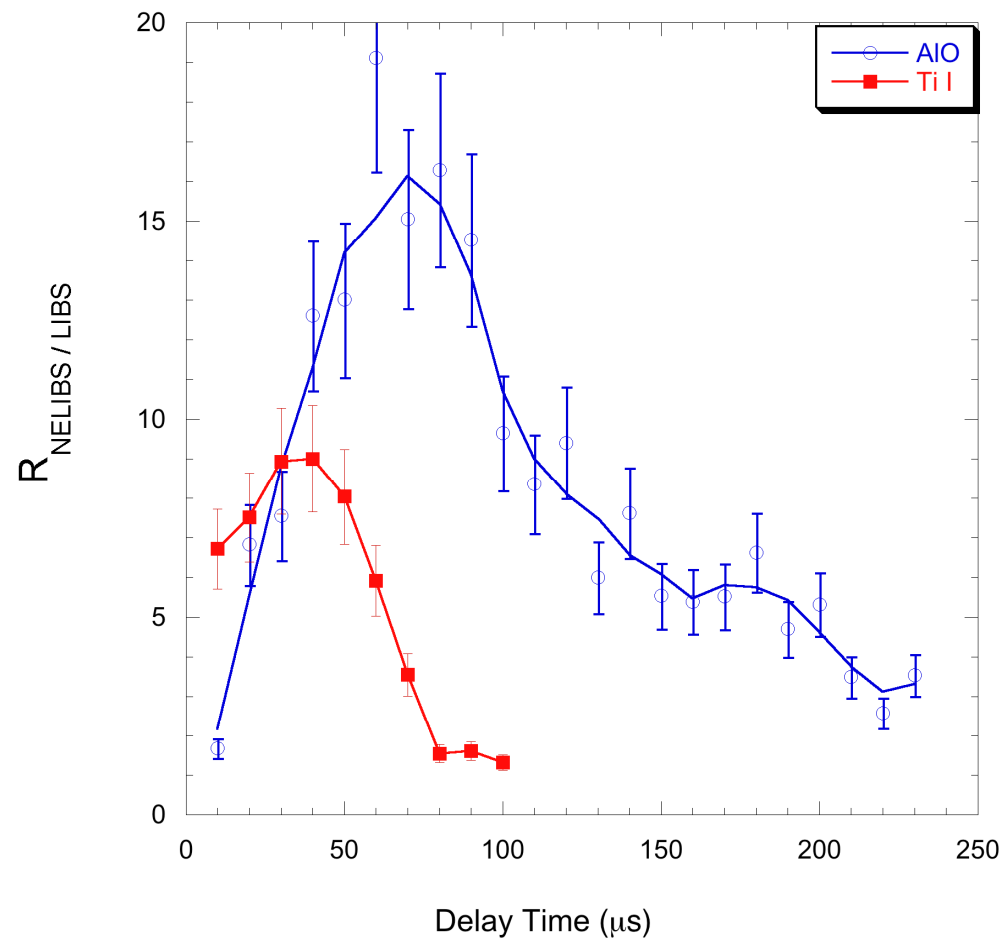

b)

FIG.5a-b 

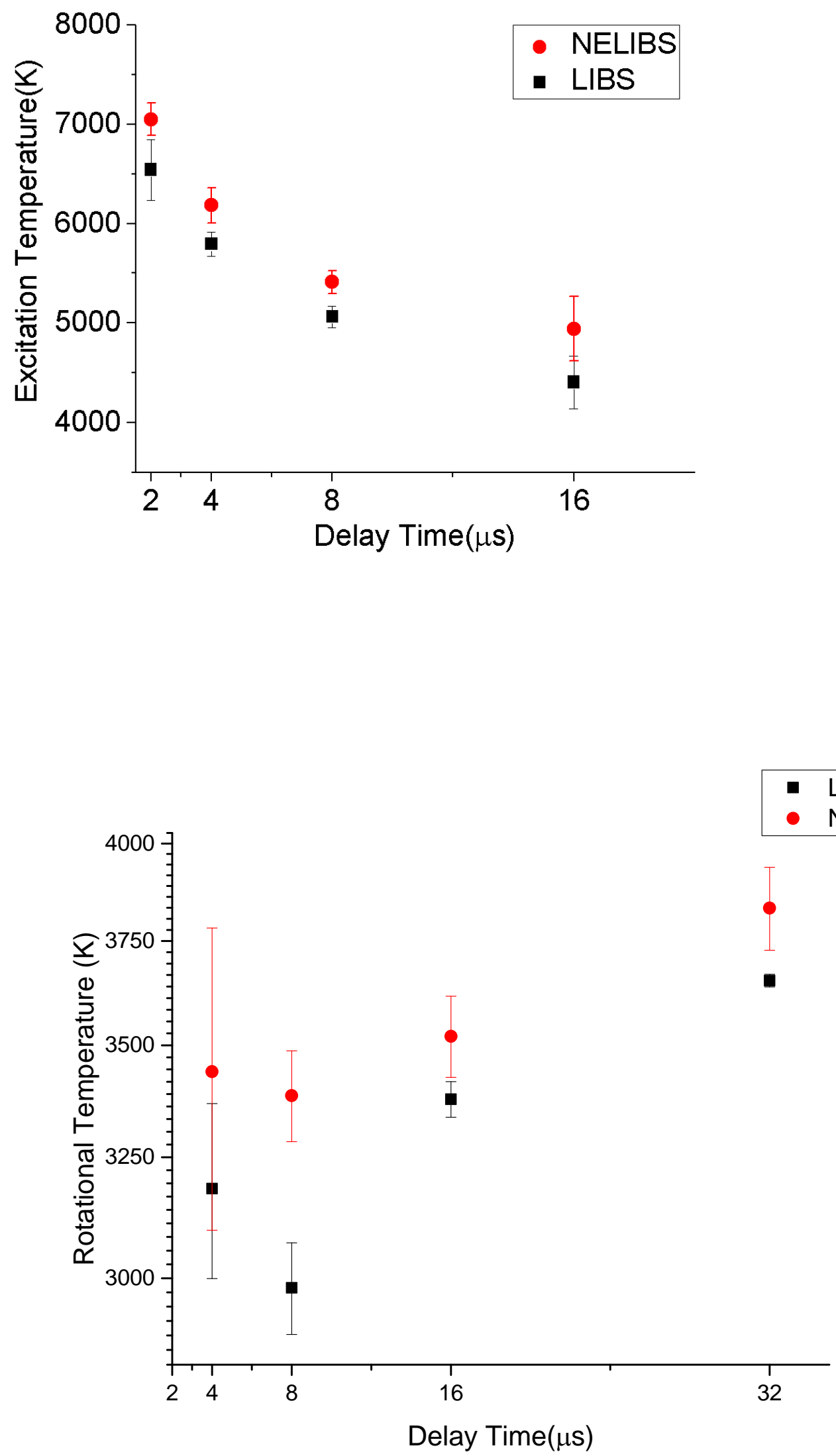

FIG.6a-b 

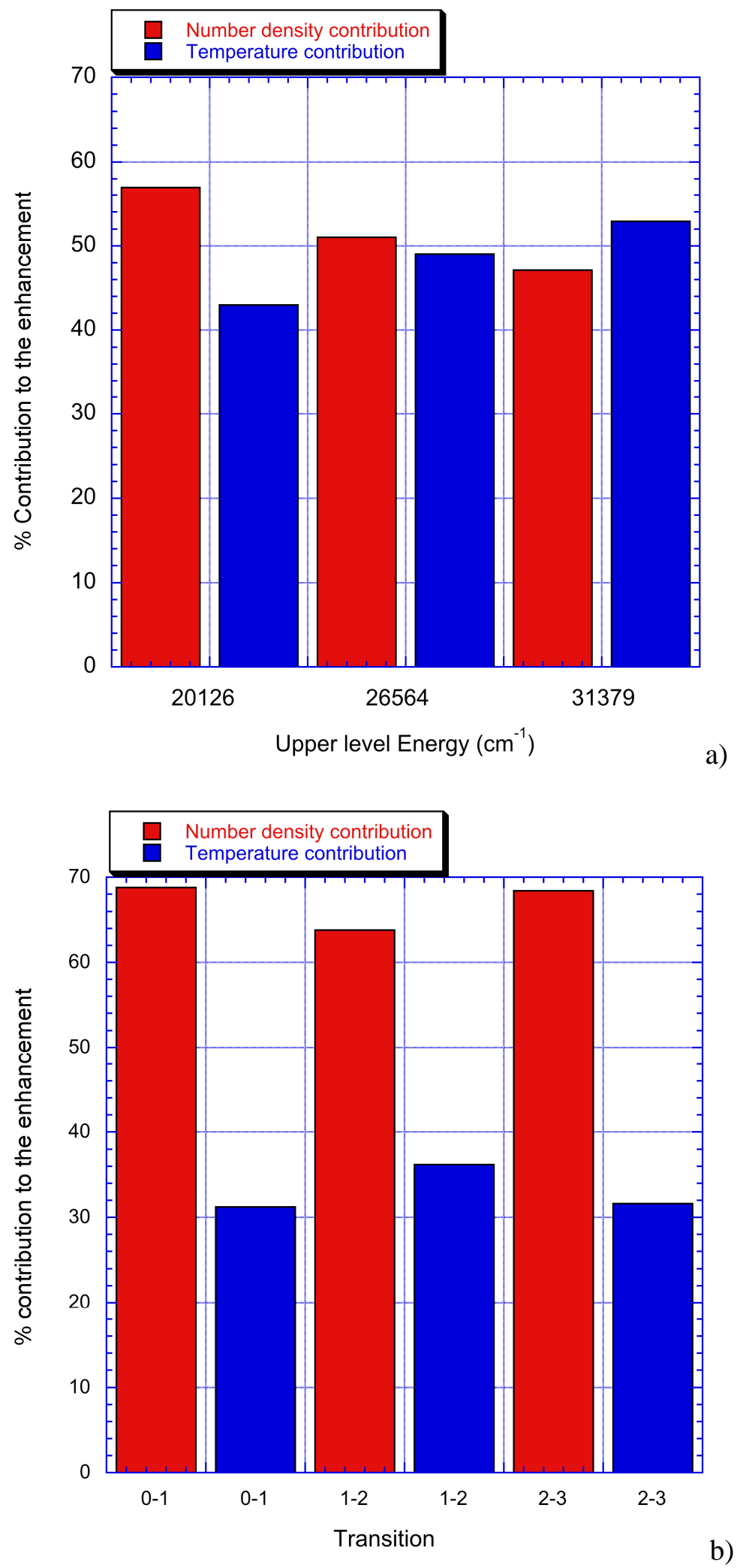

FIG.7a-b 


\begin{tabular}{|l|l|l|c|c|}
\hline Delay (ns) & NELIP Tex $(\mathrm{K})$ & LIP Tex $(\mathrm{K})$ & $\begin{array}{l}\text { NELIP } \\
\text { K·NTOT }\left(\mathrm{cm}^{-3}\right)\end{array}$ & $\begin{array}{l}\text { LIP } \\
\left.\text { K·NTOT( } \mathrm{cm}^{-3}\right)\end{array}$ \\
\hline 800 & $14300 \pm 1200$ & $10500 \pm 900$ & $7.910^{18}$ & $6.510^{16}$ \\
\hline 1300 & $8700 \pm 900$ & $10400 \pm 900$ & $8.010^{18}$ & $3.810^{16}$ \\
\hline 1800 & $8400 \pm 800$ & $10400 \pm 900$ & $5.510^{18}$ & $2.410^{16}$ \\
\hline
\end{tabular}

TAB. 1 
